1. Prager, R., Wallace, P., and Olefsky, J.M. 1987. Direct and indirect effects of insulin to inhibit hepatic glucose output in obese subjects. Diabetes. 36:607-611.

2. Sindelar, D.K., Balcom, J.H., Chu, C.A., Neal, D.W., and Cherrington, A.D. 1996. A comparison of the effects of selective increases in peripheral or portal insulin on hepatic glucose production in the conscious dog. Diabetes. 45:1594-1604.

3. Sindelar, D.K., et al. 1997 . The role of fatty acids in mediating the effects of peripheral insulin on hepatic glucose production in the conscious dog. Diabetes. 46:187-196.

4. Ader, M., and Bergman, R.N. 1990. Peripheral effects of insulin dominate suppression of fasting hyperglycemia. Am. J. Physiol. 258:E1029-E1032.

5. Giacca, A., et al. 1992. Importance of peripheral insulin levels for insulin-induced suppression of glucose production in depancreatized dogs. J. Clin. Invest. 90:1769-1777

6. Ito, K., et al. 1995. Exogenous insulin dose-dependently suppresses glucopenia-induced glucagon secretion from perfused rat pancreas. Metabolism. 44:358-362.
7. Sindelar, D.K., et al. 1998. Basal hepatic glucose production is regulated by the portal vein insulin concentration. Diabetes. 47:523-529.

8. Obici, S., Zhang, B.B., Karkanias, G., and Rossetti, L. 2002. Hypothalamic insulin signaling is required for inhibition of glucose production. Nat. Med. 8:1376-1382.

9. Davis, S.N., et al. 1995. Evidence that the brain of the conscious dog is insulin sensitive. J. Clin. Invest. 95:593-602.

10. la Fleur, S.E., Kalsbeek, A., Wortel, J., and Buijs, R.M. 2000. Polysynaptic neural pathways between the hypothalamus, including the suprachiasmatic nucleus, and the liver. Brain Res. 871:50-56.

11. Cherrington, A.D., et al. 2002. Metabolic importance of portal insulin delivery. Diabetes. 51(Suppl. 2):A313.

12. Fisher, S.J., and Kahn, C.R. 2003. Insulin signaling is required for insulin's direct and indirect action on hepatic glucose production. J. Clin. Invest. 111:463-468. doi:10.1172/JCI200316426.

13. Buettner, C., et al. 2005. Severe impairment in liver insulin signaling fails to alter hepatic insulin action in conscious mice. J. Clin. Invest. 115:1306-1313. doi:10.1172/JCI200523109.

14. Okamoto, H., Obici, S., Accili, D., and Rossetti, L. 2005. Restoration of liver insulin signaling in Insr knockout mice fails to normalize hepatic insulin action. J. Clin. Invest. 115:1314-1322. doi:10.1172/ JCI200523096.

15. Duckworth, W.C., Bennett, R.G., and Hamel, F.G. 1997. The significance of intracellular insulin to insulin action. J. Invest. Med. 45:20-27.

16. Duckworth, W.C., Bennett, R.G., and Hamel, F.G. 1998. Insulin acts intracellularly on proteasomes through insulin-degrading enzyme. Biochem. Biophys. Res. Comm. 244:390-394.

17. Lewis, G.F., Zinman, B., Groenewoud, Y., Vranic, M., and Giacca, A. 1996. Hepatic glucose production is regulated both by direct hepatic and extrahepatic effects of insulin in humans. Diabetes. 45:454-462.

18. Cherrington, A.D., Edgerton, D., and Sindelar, D.K. 1998. The direct and indirect effects of insulin on hepatic glucose production. Diabetologia. 41:987-996.

\title{
Contribution of adipose tissue and de novo lipogenesis to nonalcoholic fatty liver disease
}

\author{
Shinji Tamura and lichiro Shimomura
}

Department of Internal Medicine and Molecular Science, Graduate School of Medicine, Osaka University, Suita, Osaka, Japan.

\begin{abstract}
Nonalcoholic fatty liver disease (NAFLD) is a component of the metabolic syndrome, with a clinical spectrum ranging from simple fatty liver to steatohepatitis, cirrhosis, and hepatocellular carcinoma. The primary event of NAFLD is the accumulation of triacylglycerols (TAGs) in hepatocytes. In this issue of the JCI, Donnelly et al. report on their use of stable isotope methodology to show that fatty acids stored in adipose tissue and fatty acids newly made within the liver through de novo lipogenesis are the major sources of TAGs in the liver and are secreted as lipoproteins in NAFLD (see the related article beginning on page 1343).
\end{abstract}

Nonalcoholic fatty liver disease (NAFLD) is the most common cause of abnormal results in liver-function tests. This clinical situation is really a continuum of diseases that includes simple (benign) fatty liver, nonalcoholic steatohepatitis (NASH), cirrhosis, and hepatocellular carcinoma (1). NAFLD is defined as liver steatosis in patients who do not consume enough alcohol to cause hepatic injury. Although some drugs or genetic abnormalities can cause NAFLD, the majority of cases are associated with obesity, insulin resistance,

Nonstandard abbreviations used: ChREBP, carbohydrate responsive element-binding protein; DNL, de novo lipogenesis; NAFLD, nonalcoholic fatty liver disease; NASH, nonalcoholic steatohepatitis; NEFA, nonesterified fatty acid; TAG, triacylglycerol.

Conflict of interest: The authors have declared that no conflict of interest exists.

Citation for this article: J. Clin. Invest. 115:1139-1142 (2005). doi:10.1172/JCI200524930. and type 2 diabetes. In order to clarify the pathogenesis of this disease and identify potential therapeutic targets, an increased understanding of the dynamics of triacylglycerol (TAG) metabolism in the liver in relation to whole-body metabolic status is needed. Sources of hepatic TAGs include dietary TAGs that are transported via chylomicrons from the intestine to adipose tissue or to the liver, where they are secreted via lipoproteins, in addition to TAGs that are synthesized from fatty acids and glycerol in the liver. Fatty acids required for TAG synthesis are available from both the plasma nonesterified fatty acid (NEFA) pool and the pool of fatty acids newly synthesized within the liver through de novo lipogenesis (DNL). TAGs present in the liver may be stored as lipid droplets or secreted into the blood as VLDLs; they may also enter the oxidation pathway (Figure 1). The study by Don- nelly et al. in this issue of the JCI examines hepatic lipoprotein metabolism in humans and describes the major sources of hepatic and plasma lipoprotein TAG in NAFLD (2).

\section{The NEFA pool}

Donnelly et al. found that the plasma NEFA pool accounts for approximately $60 \%$ of TAG content in the livers of NAFLD patients, which reflects the importance of the NEFA pool in the pathogenesis of NAFLD (2). The hepatic uptake of fatty acids is not regulated and, as a result, plasma fatty acid concentration is directly related to the influx of fatty acids to the liver. The authors report that, in the fasted state, adipose tissue contributes approximately $80 \%$ of fatty acid content to the plasma NEFA pool, and even in the fed state, this contribution remains at approximately $60 \%$. Thus, the overproduction of fatty acids in adipose tissues that flow to the liver via the NEFA pool is the most likely explanation for excess TAG accumulation in NAFLD. In insulin-resistant states, insulin does not fully suppress the activity of hormone-sensitive lipase, which catalyzes the hydrolytic release of fatty acids from the TAG moiety and results in enhanced lipolysis and flux of fatty acids to the plasma NEFA pool (Figure 1). In addi- 


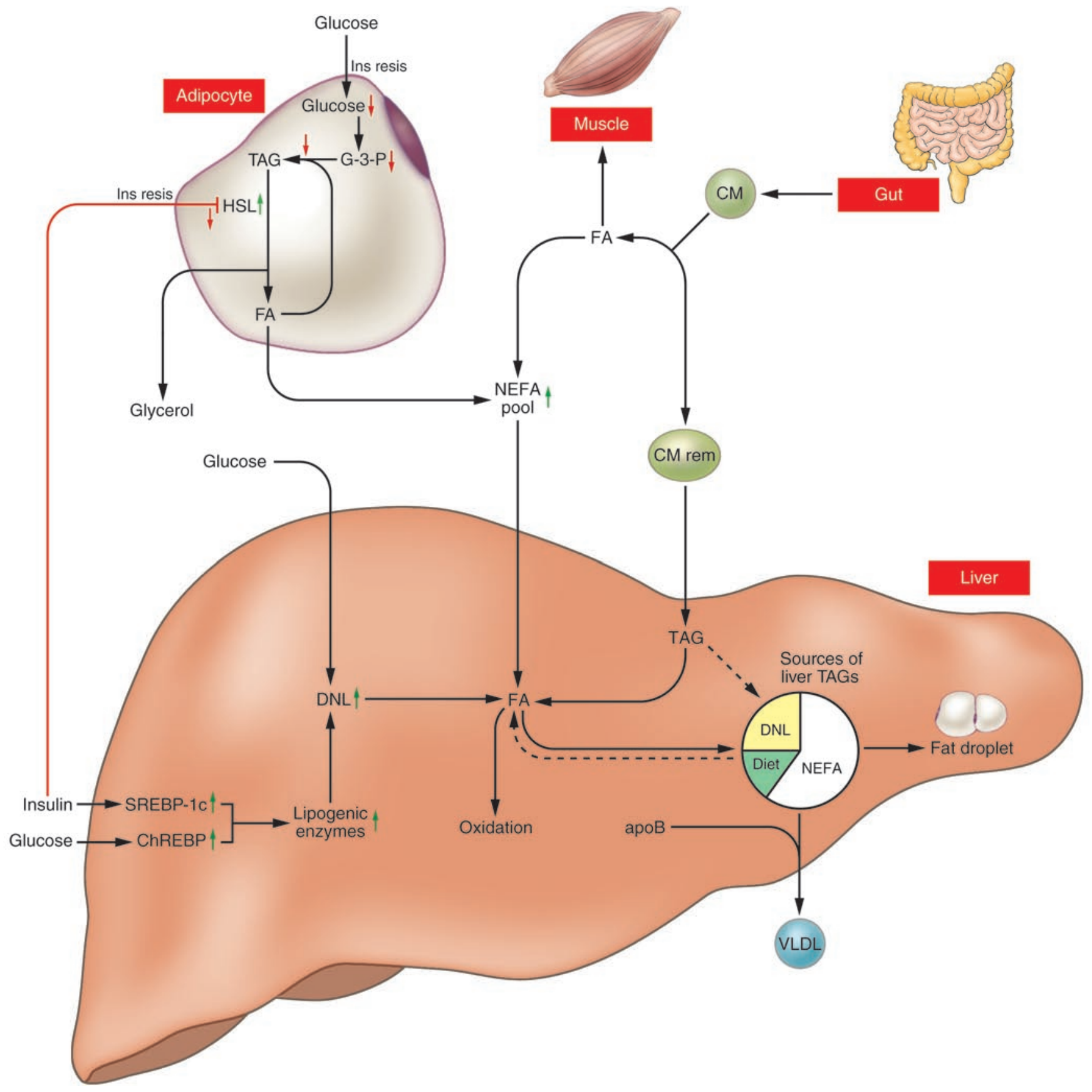

Figure 1

Altered TAG metabolism in NAFLD. Insulin resistance causes insufficient suppression of hormone-sensitive lipase (HSL) activity and reduced glucose uptake in adipose tissue, resulting in enhanced lipolysis and increased fatty acid flux into the plasma NEFA pool. In this issue of the $\mathrm{JCl}$, Donnelly et al. report that approximately $60 \%$ of TAGs accumulated in the liver are derived from the plasma NEFA pool (2). Hyperinsulinemia and hyperglucosemia induce SREBP-1c and ChREBP in the liver, respectively. Both of these transcription factors activate most genes required for lipogenesis, which enhances DNL in the liver. Approximately $25 \%$ of accumulated TAGs in the liver are derived from DNL (2). CM, chylomicron; CM rem, CM remnant; FA, fatty acid; G-3-P, glycerol-3-phosphate; Ins resis, insulin resistance.

tion, reduced glucose uptake due to insulin resistance reduces glycerol-3-phosphate levels, thereby reducing the reutilization of fatty acids for TAG synthesis (Figure 1). Thus, the insulin resistance in adipose tissue is important as a pathogenic factor of
NAFLD. Recently, dysregulated adipocytokines such as adiponectin and TNF- $\alpha$ have been examined as causative candidates of insulin resistance (3), and it was recently reported that oxidative stress in accumulated fat causes dysregulated adipocytokine production (4). In light of these findings, it seems possible that the reduction of oxidative stress as well as the use of insulin-sensitizing agents such as thiazolidinediones and metformin may prove to be successful treatments for NAFLD. 
Contrary to the results reported here, Diraison et al. reported that the uptake of plasma NEFAs by the liver was not increased in NAFLD (5). However, Diraison et al. acknowledged that the rate of NEFA flux to the liver may have been underestimated due to the entry of plasma NEFAs into the liver via portal flow from visceral adipose tissue. Thus, in order to establish the contribution of NEFAs to TAG accumulation in the liver, the actual rate of NEFA uptake in the liver in NAFLD must be clarified.

\section{De novo lipogenesis}

In healthy human subjects, the contribution of DNL in the liver to TAG content in the fasted state is very small (less than $5 \%$ for VLDL-TAG) (6). It has been reported that DNL in the liver is elevated in insulin-resistant states and in NAFLD (7). Donnelly et al. found that DNL accounted for $26 \%$ of liver TAG content in hyperinsulinemic subjects with NAFLD (2). In healthy subjects, DNL is elevated following meals (23\% for VLDL-TAG) (6), which can be accounted for by elevation in the circulating levels of lipogenesis precursors. However, in NAFLD, DNL was already elevated in the fasted state, and further postprandial elevation was not observed. Indeed, constant elevation of DNL was also observed in subjects fed a diet high in simple carbohydrates for 25 days (8). These observations reflect the sustained elevation of enzymes involved in hepatic DNL.

The regulation of enzymes involved in human lipogenesis can be precisely studied by experiments in animals. Insulin activates the membrane-bound transcription factor SREBP-1c, which transcriptionally activates most genes required for lipogenesis. In mice, even in the insulinresistant state, insulin stimulates hepatic SREBP-1c transcription and increases lipogenesis (9). Lipogenesis is also regulated by glucose: glucose activates the carbohydrate response element-binding protein (ChREBP), which induces gene expression of liver-type pyruvate kinase, a key regulatory enzyme in glycolysis; this enzyme in turn provides the precursors for lipogenesis (10). ChREBP also stimulates gene expression of most enzymes involved in lipogenesis (11). Hyperinsulinemia and hyperglycemia may also induce these transcriptional factors in humans (Figure 1), although this remains to be directly demonstrated.

\section{Other factors}

Mice with peripheral insulin resistance due to adipose- and muscle-specific GLUT4 double knockout showed increased DNL accompanied by hepatic elevation of SREBP-1c and acetyl-CoA carboxylase (12). However, in these mice, increased DNL did not result in increased TAG level in the liver, nor did it increase steady-state serum levels of TAGs or NEFAs; this may be the result of a compensatory enhancement of peripheral fatty acid usage. Unlike in this animal model, such compensation is assumed to be insufficient in human subjects with type 2 diabetes (13). The reduced use of fatty acids in peripheral tissues in combination with elevated DNL may contribute to TAG accumulation in the livers of subjects with NAFLD. Muscle exercise is effective in reducing TAG accumulation in the liver not only by improving insulin resistance but also by enhancing peripheral fatty acid disposal.

The accumulation of TAGs in the liver results from an imbalance among the uptake, synthesis, export, and oxidation of fatty acids. In type 2 diabetes, secretion of VLDL is increased due to insulin resistance (14). It has been reported, however, that in NAFLD, VLDL secretion is not increased compared with secretion in controls (5). Because of methodological differences, these results cannot be directly compared. Secretion rates of hepatic VLDL should be quantified in hyperinsulinemic subjects with and without hepatic steatosis. $\beta$-oxidation of fatty acids in the liver was found to be increased in patients with NASH (15). However, the increase is not sufficient for overcoming elevated rates of hepatic TAG synthesis. The increase in NEFA oxidation may account for the apparent oxidative stress that causes hepatic injury in NAFLD.

In the study by Donnelly et al. (2), considerable variation was present in liver TAG level as well as in the degrees of inflammation and fibrosis in the liver. In some cases of NASH, the amount of accumulated fat in the liver decreased as the disease progressed to cirrhosis (16). In addition, the degree of liver damage may influence lipid metabolism and apparent insulin sensitivity $(15,17)$. Thus, to clarify the earliest events in TAG accumulation precisely, metabolic analysis of patients with simple fatty liver (i.e., without hepatitis) is needed.

Donnelly et al. (2) show that the contributions of fatty acid sources of TAG in VLDL are almost the same as those for the TAG pool in the liver. Although these findings result from studies of NAFLD patients, the approach used will allow us to further investigate this in vivo biochemistry at various stages in the development of NAFLD, as well as the effects of diet, exercise, and pharmacological therapies on the disease.

Address correspondence to: Shinji Tamura, Department of Internal Medicine and Molecular Science, Graduate School of Medicine, Osaka University, 2-2 B5, Yamada-oka, Suita, Osaka 565-0871, Japan. Phone: 81-6-6879-3732; Fax: 81-66879-3739; E-mail: tamuras@imed2.med. osaka-u.ac.jp.

1. Tolman, K.G., Fonseca, V., Tan, M.H., and Dalpiaz, A. 2004. Narrative review: hepatobiliary disease in type 2 diabetes mellitus. Ann. Intern. Med. 141:946-956.

2. Donnelly, K.L., et al. 2005. Sources of fatty acids stored in liver and secreted via lipoproteins in patients with nonalcoholic fatty liver disease. J. Clin. Invest. 115:1343-1351. doi:10.1172/JCI200523621.

3. Maeda, N., et al. 2002. Diet-induced insulin resistance in mice lacking adiponectin/ACRP30. Nat. Med. 8:731-737.

4. Furukawa, S., et al. 2004. Increased oxidative stress in obesity and its impact on metabolic syndrome. J. Clin. Invest. 114:1752-1761. doi:10.1172/ JCI200421625.

5. Diraison, F., Moulin, P., and Beylot, M. 2003. Contribution of hepatic de novo lipogenesis and reesterification of plasma non esterified fatty acids to plasma triglyceride synthesis during non-alcoholic fatty liver disease. Diabetes Metab. 29:478-489.

6. Timlin, M.T., and Parks, E.J. 2005. Temporal pattern of de novo lipogenesis in the postprandial state in healthy men. Am. J. Clin. Nutr. 81:35-42.

7. Schwarz, J.-M., Linfoot, P., Dare, D., and Aghajanian, K. 2003. Hepatic de novo lipogenesis in normoinsulinemic and hyperinsulinemic subjects consuming high-fat, low-carbohydrate and low-fat, high-carbohydrate isoenergetic diets. Am. J. Clin. Nutr. 77:43-50.

8. Hudgins, L.C., et al. 1996. Human fatty acid synthesis is stimulated by a eucaloric low fat, high carbohydrate diet. J. Clin. Invest. 97:2081-2091.

9. Shimomura, I., Bashmakow, Y., and Horton, J.D. 1999. Increased levels of nuclear SREBP-1c associated with fatty livers in two mouse models of diabetes mellitus. J. Biol. Chem. 274:30028-30032.

10. Yamashita, H., et al. 2001. A glucose-responsive transcription factor that regulates carbohydrate metabolism in the liver. Proc. Natl. Acad. Sci. U. S. A. 98:9116-9121.

11. Iizuka, K., Bruick, R.K., Liang, G., Horton, J.D., and Uyeda, K. 2004. Deficiency of carbohydrate response element binding protein (ChREBP) reduces lipogenesis as well as glycolysis. Proc. Nat. Acad. Sci. U. S. A. 101:7281-7286.

12. Kotani, K., Peroni, O.D., Minokoshi, Y., Boss, O., and Kahn, B.B. 2004. GLUT4 glucose transporter deficiency increases hepatic lipid production and peripheral lipid utilization. J. Clin. Invest. 114:1666-1675. doi:10.1172/JCI200421341.

13. Kelly, D.E., and Mandarino, L.J. 2000. Fuel selection in human skeletal muscle in insulin resistance: a reexamination. Diabetes. 49:677-683.

14. Cummings, M.H., et al. 1995. Increased hepatic secretion of very-low-density lipoprotein 
apolipoprotein B-100 in NIDDM. Diabetologia. 38:959-967.

15. Sanyal, A.J., et al. 2001. Nonalcoholic steatohepatitis: association of insulin resistance and mitochondrial abnormalities. Gastroenterology.
120:1183-1192.

16. Powell, E.E., et al. 1990. The natural history of nonalcoholic steatohepatitis: a follow-up study of forty-two patients for up to 21 years. Hepatology. 11:74-80.
17. Schattenberg, J.M., Wang, Y., Singh, R., Rigoli, R.M., and Czaja, M.J. 2005. Hepatocyte CYP2E1 overexpression and steatohepatitis lead to impaired hepatic insulin signaling. J. Biol. Chem. 280:9887-9894.

\title{
Caffey disease: an unlikely collagenopathy
}

\author{
Francis H. Glorieux
}

Departments of Surgery, Pediatrics, and Human Genetics, McGill University, and Genetics Unit, Shriners Hospital for Children, Montréal, Quebec, Canada.

Infantile cortical hyperostosis (also known as Caffey disease) is characterized by hyperirritability, acute inflammation of soft tissues, and profound alterations of the shape and structure of the underlying bones, particularly the long bones, mandible, clavicles, or ribs. In this issue of the JCI, Gensure et al. undertook fine mapping of the genetic locus for this disease in a large kindred of individuals with the autosomal dominant form of the condition. The authors found a novel missense mutation in COL1A1, the gene encoding the $\alpha 1$ chain of type I collagen, in all affected individuals in 3 discrete pedigrees (see the related article beginning on page 1250). This is a surprising finding, as all other reported mutations affecting the synthesis of type I collagen lead to conditions such as osteogenesis imperfecta and Ehlers-Danlos syndrome, in which quantitative or qualitative defects in type I collagen synthesis give rise to bone fragility and/or connective tissue hyperextensibility. The deleterious effect of the mutation on collagen fibril morphology is demonstrated; however, the precise functional link between the reported missense mutation and the localized inflammation and hyperostosis seen in Caffey disease awaits future studies.

Infantile cortical hyperostosis (ICH) - also referred to as Caffey or Caffey-Silverman disease - was recognized in 1945 by Caffey and Silverman (1). The condition, later shown to be transmitted as an autosomal dominant trait with incomplete penetrance, becomes clinically evident before 5-7 months of life, and the average age at onset is around 9 weeks. The initial symptom is general hyperirritability, with fever and anorexia, quickly followed by painful, firm soft-tissue swelling, particularly in the face (cheeks and jaws), the scapular region, and upper and lower limbs. It is usually self-limited, with severe symptoms lasting from 2-3 weeks to 2-3 months. The pattern of distribution of the lesions varies from patient to patient and can be symmetrical (Figure 1). Swelling also involves underlying muscle. It is important to

Nonstandard abbreviations used: COL1A1, gene encoding the $\alpha 1$ chain of type I collagen; EDS, EhlersDanlos syndrome; ICH, infantile cortical hyperostosis; PGE, prostaglandin E.

Conflict of interest: The author has declared that no conflict of interest exists.

Citation for this article: J. Clin. Invest. 115:1142-1144 (2005). doi:10.1172/JCI200525148. note that this phase of acute inflammation precedes the abnormal thickening of cortical bone (hyperostosis) that gives its name to the disease. It also subsides long before the hyperostosis resolves. Sometimes lesions recur suddenly in their original sites or in new sites, either during or after the subsidence of the swellings that appeared at the onset of the disease. This uneven protracted clinical course with unpredictable remissions and relapses is one of the most characteristic features of the condition (2).
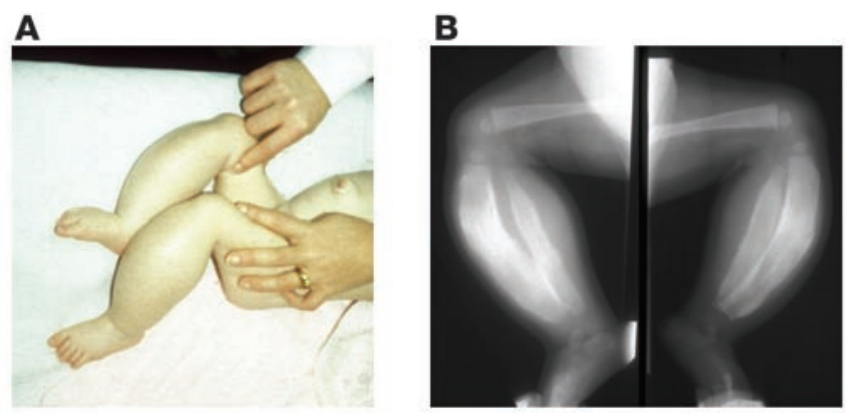

Figure 1

A 6-month-old girl with typical clinical findings of Caffey disease, which appeared at age 7 weeks. The painful swelling of soft tissue around the legs $(\mathbf{A})$ is matched by severe involvement of the tibia and fibula bilaterally (x-radiography, B). Femurs are strikingly unaffected. 